\title{
Sorotipos de Actinobacillus pleuropneumoniae isolados no Brasil de 1993 a 2006
}

\author{
Serotypes of Actinobacillus pleuropneumoniae isolated in Brazil from 1993 to 2006 \\ Suzana Satomi Kuchiishi' ${ }^{1}$, Jalusa Deon Kich ${ }^{2}$, Marni Lúcia Fracasso Ramenzoni², Denis \\ Spricigo $^{3}$, Catia Silene Klein ${ }^{2}$, Maria Bernardete Burin Fávero ${ }^{2}$ \& Itamar Antônio Piffer ${ }^{4}$
}

\begin{abstract}
RESUMO
A pleuropneumonia suína (PPS) é uma doença infecto-contagiosa decorrente da infecção e efeitos patológicos do Actinobacillus (A.) pleuropneumoniae, caracterizada por pneumonia necrótica e hemorrágica associada à pleurisia fibrinosa. A doença causa prejuízos devido a perdas por morte, pouco desenvolvimento e condenações de carcaças no abatedouro. $\mathrm{O}$ isolamento do A. pleuropneumoniae é necessário para investigação de resistência a antibióticos e a identificação do sorotipo é importante para estudos epidemiológicos e controle da doença através de programas de vacinações. Este trabalho mostra a ocorrência dos sorotipos da bactéria através da sorotipificação pela composição da cápsula polissacarídea por imunodifusão em gel de agarose contra soros dos sorotipos 1 ao 12. As amostras (399) analisadas foram isoladas no período de 1993 a 2006 em diferentes regiões do Brasil. Houve 171 amostras não sorotipificáveis. Durante o período estudado, os sorotipos mais prevalentes foram 5 (14,8\%), 3 (13,8\%), 10 (7,3\%),6 (6,5\%) e 7 (5\%). Foi observado diminuição na ocorrência dos sorotipos 5 e 3 a partir do ano de 2000, indicando que as vacinas empregadas possivelmente diminuíram a doença clínica. Neste tempo, outros sorotipos como o 4, 6, 8 e 10 ocorreram, o que requer maior atenção no comércio de animais, assim como o monitoramento dos rebanhos brasileiros.
\end{abstract}

Descritores: Actinobacillus pleuropneumoniae, pleuropneumonia suína, sorotipificação, antígeno capsular.

\section{ABSTRACT}

Porcine pleuropneumonia is an infect-contagious disease caused by infection and pathological effects of Actinobacillus (A.) pleuropneumoniae, characterized by necrotic and hemorrhagic pneumonia associated to the fibrinous pleurisy. The disease causes economic losses from death, lower weight gain and carcasses depreciation in the slaughterhouse. A. pleuropneumoniae isolation is necessary for antibiotic resistance investigation and serotyping, that is important for epidemiological studies and control of the disease by vaccination programs. This work shows the occurrence of $A$. pleuropneumoniae serotypes by the polysaccharide capsule identification using immunodiffusion in agarosis gel with serum against serotypes 1 to 12 . The samples (399) analyzed were isolated from 1993 to 2006 at different Brazilian's regions. There was 171 (42,9\%) samples that were no serotyping. During the studied time the most prevalent serotypes were $5(14,8 \%), 3(13,8 \%), 10(7,3 \%), 6(6,5 \%)$ and $7(5 \%)$. A decrease in the occurrence of the serotypes 5 and 3 was observed from 2000, indicating that the vaccination programs possibly reduced the clinical disease. In this time other serotypes 4,6,8 and 10 occurred, that requests larger attention in the trade of animals as well as the monitoring of the Brazilian herds.

Key words: Actinobacillus pleuropneumoniae, swine pleuropneumonia, serotyping, capsular antigen. 


\section{INTRODUÇÃO}

A pleuropneumonia suína (PPS) é distribuída mundialmente e sua importância é maior nas regiões de produção intensiva de suínos. As perdas econômicas ocorrem devido à piora na conversão alimentar, mortalidade, gastos com medicamentos, vacinações e condenação de vísceras e carcaças no abatedouro. No Brasil, a PPS foi diagnosticada pela primeira vez em Santa Catarina em 1981 [8]. A partir de então vários surtos desta doença foram diagnosticados no sul do Brasil [13].

O A. pleuropneumoniae, a bactéria causadora da PPS possui até o momento 15 sorotipos descritos, sendo que a diferença entre eles é determinada pela composição da cápsula polissacarídea que cobre a parede celular. Com base nessa informação, 12 sorotipos foram anteriormente classificados para o biotipo 1 que requer nicotina amida adenina dinucleotídeo (NAD) para o seu crescimento [10]. Os sorotipos 13 e 14 são NAD independentes, biotipo 2 e o último sorotipo descrito foi o 15 que também é NAD dependente [1,11].

O objetivo do presente trabalho foi mostrar a ocorrência dos sorotipos de Actinobacillus pleuropneumoniae de amostras isoladas no Brasil através da sorotipificação pela composição da cápsula polissacarídea por imunodifusão em gel de agarose no período de 1993 a 2006.

\section{MATERIAIS E MÉTODOS}

As amostras de A. pleuropneumoniae foram isoladas de material proveniente de nódulos pulmonares, biopsia de tonsila, suabes de cavidade nasal ou cultura enviada a Embrapa Suínos e Aves para diagnóstico e sorotipificação. O A. pleuropneumoniae cresce em agar sangue com 5\% de sangue ovino com estria perpendicular de Staphylococcus aureus, fornecedora de NAD, produzindo hemólise. As colônias NAD-dependentes foram submetidas à caracterização bioquímica e classificação das espécies, conforme métodos e tabelas já descritas [6,9]. A técnica utilizada para sorotipagem foi a imunodifusão com extração fenólica de antígeno capsular contra os soros hiperimunes dos 12 sorotipos padrões (1 ao 12) de A. pleuropneumoniae [12]. O sorotipo 15 não foi incluído neste trabalho. Os soros foram produzidos em coelhos e aqueles que produziram reação cruzada foram absorvidos com um sorotipo heterólogo, com o intuito de remover anticorpos comuns à espécie [5]. De 1993 a 2006 foram sorotipificados 399 amostras.

\section{RESULTADOS}

Na Tabela 1 encontram-se os resultados das sorotipificações realizadas a cada ano desde 1993 a 2006 em várias regiões do Brasil.

\section{DISCUSSÃO}

A sorotipificação do A. pleuropneumoniae no Brasil iniciou-se na década de 80. De 1981 a 1993 foram sorotipificadas 55 amostras de A. pleuropneumoniae, das quais 30 pertenciam ao sorotipo 5 , quinze ao sorotipo 3 e sete ao sorotipo 7 [14]. Os autores demonstraram a maior prevalência do sorotipo 5 no Brasil, seguido do 3 e 7 e um aumento da ocorrência do sorotipo 3 considerado menos virulento, cuja disseminação pode ter ocorrido pelo comércio de animais. Houve um aumento do número de sorotipos presentes no país, de 1981-1985 foram diagnosticados os sorotipos 5 e 7, de 1986-1989, os sorotipos 3, 5 e 7, de 1990-1993, os sorotipos 1, 3, 5, 7 e 9 e no presente trabalho de 1993-2006 a ocorrência dos sorotipos 1, 3, $4,5,6,7,8,10,11$ e 12 conforme a Figura 1, ou seja, neste período, a ocorrência de sorotipos que ainda não haviam sido relatados no Brasil (sorotipos 4, 8 e 11). Em 1999 isolou-se pela primeira vez no Brasil o sorotipo 12 e no mesmo ano através de soroaglutinação rápida em placa, demonstrou-se a ocorrência do sorotipo 6 (9/24) [3,7]. Em 2002, houve o primeiro relato de surto pelo sorotipo $10 \mathrm{em}$ Minas Gerais, a partir de então ocorreu um aumento de casos de PPS causados por este sorotipo [4]. Nota-se que até 1999, era grande o número de isolamentos dos sorotipos 5 e 3 , mas que de 2000 em diante houve uma considerável diminuição, enquanto que outros sorotipos apareceram e aumentaram sua importância. $\mathrm{O}$ que pode ter acontecido é que as vacinas conseguiram diminuir a doença clínica nos rebanhos que era causada pelos sorotipos 3 e 5, oportunizando o diagnóstico de outros sorotipos. Somando todas as amostras, observa-se que o sorotipo 5 ainda é o mais prevalente (59/399) seguido dos sorotipos 3, 10, 6, 7, 4 e 8. Em todo o período, houve 171 amostras não sorotipáveis, fato que pode ter ocorrido devido a algumas amostras perderem a capacidade de produzir cápsula, por limitação do método utilizado, presença do sorotipo 15 ou outros sorotipos ainda não descritos. Esses resultados demonstram a dinâmica epidemiológica entre os sorotipos no Brasil e chamam a atenção para a necessidade de identifica- 
Tabela 1. Número de amostras sorotipificadas de Actinobacillus pleuropneumoniae através de imunodifusão em agarose durante o período de 1993 a 2006 em diferentes regiões do Brasil.

\begin{tabular}{|c|c|c|c|c|c|c|c|c|c|c|c|c|c|c|c|}
\hline & 1993 & 1994 & 1995 & 1996 & 1997 & 1998 & 1999 & 2000 & 2001 & 2002 & 2003 & 2004 & 2005 & 2006 & Total \\
\hline s1 & - & 2 & - & - & - & - & 2 & - & 3 & - & - & - & - & - & 7 \\
\hline S2 & - & - & - & - & - & - & - & - & - & - & - & - & - & - & 0 \\
\hline S3 & 2 & 10 & 18 & 1 & 2 & 1 & - & - & - & - & 1 & - & 4 & 16 & 55 \\
\hline S4 & - & - & - & 8 & - & - & - & 1 & 1 & 1 & - & - & 2 & - & 13 \\
\hline S5 & 27 & 7 & 1 & - & - & - & - & 1 & - & 5 & 7 & 7 & 3 & 1 & 59 \\
\hline S6 & - & - & - & - & - & - & - & - & - & - & 5 & 18 & 2 & 1 & 26 \\
\hline S7 & 5 & - & 2 & - & - & - & - & 2 & 1 & 1 & - & 1 & - & 8 & 20 \\
\hline S8 & - & - & - & - & - & - & - & - & - & - & - & 12 & - & - & 12 \\
\hline s9 & - & - & - & - & - & - & - & - & - & - & - & - & - & - & 0 \\
\hline S10 & - & - & - & - & - & - & - & - & - & 3 & - & 8 & 11 & 7 & 29 \\
\hline S11 & - & 1 & - & - & - & - & - & - & 1 & - & - & - & - & - & 2 \\
\hline S12 & - & - & 3 & 2 & - & - & - & - & - & - & - & - & - & - & 5 \\
\hline NS & 2 & - & 19 & 61 & - & 1 & 8 & 2 & 13 & 4 & 5 & 11 & 38 & 7 & 171 \\
\hline
\end{tabular}

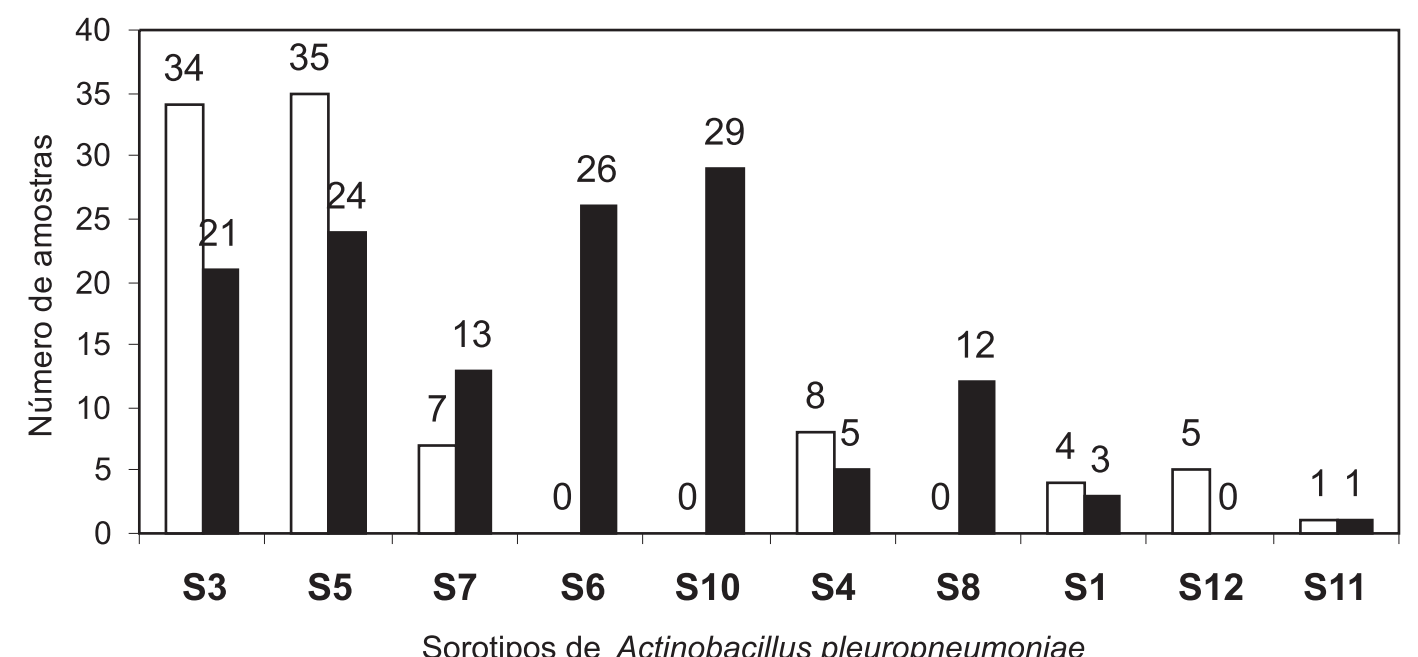

Sorotipos de Actinobacillus pleuropneumoniae

$\square$ 1993-1999 $\square$ 2000-2006

Figura 1. Ocorrência de sorotipos de Actinobacillus pleuropneumoniae durante o período de 1993-1999 e 2000-2006 no Brasil.

ção do sorotipo, pois para o controle da doença, o conhecimento dos sorotipos de A. pleuropneumoniae existentes numa região é importante, pois nem sempre as vacinas disponíveis contemplam o sorotipo presente. A monitoria de cepas novas introduzidas no rebanho, assim como infecções associadas de sorotipos podem ser limitantes à eficiência de programas de controle específicos [2].

\section{CONCLUSÕES}

O trabalho mostra o aumento da ocorrência dos sorotipos 4, 6, 8 e 10 de Actinobacillus pleuropneumoniae no Brasil, o que requer maior atenção no comércio de animais, assim como o monitoramento dos rebanhos brasileiros, demonstrando a importância da identificação do sorotipo para utilizar-se vacina correta e diminuir os efeitos da doença. 


\section{REFERÊNCIAS}

1 Blackall P.J., Klaasen H.L., Van Den Bosch H., Kuhnert P. \& Frey J. 2002. Proposal of a new serovar of Actinobacillus pleuropneumoniae: serovar 15. Veterinary Microbiology. 84 (Suppl 1): 47-52.

2 Chiers K., Donne E., Van Overbeke I., Ducatelle, R. \& Haesebrouck, F. 2002. Actinobacillus pleuropneumoniae infections in closed swine herds: infection patterns and serological profiles. Veterinary Microbiology. 85: 343-352.

3 Costa A.T.R, Reis R., Ferreira H.B.C. \& Reis F.T. 1999. Sorotipificação de Actinobacillus pleuropneumoniae isolados de lesões pulmonares de suínos. In: Resumos do IX Congresso Brasileiro de Veterinários Especialistas em Suínos (Belo Horizonte, Brasil). p.179-180.

4 Costa A.T.R, Reis R. \& Ferreira H.B.C. 2003. Isolamento e controle do Actinobacillus pleuropneumoniae (APP) sorotipo 10 de um surto de pleuropneumonia suína. In: XI Congresso Brasileiro de Veterinários Especialistas em Suínos (Goiânia, Brasil). p.29-30.

5 Gunnarson A., Biberstein E.L. \& Hurvell B. 1977. Serologic studies on porcine strains of Haemophilus parahaemolyticus (pleuropneumoniae). Agglutination reactions. American Journal of Veterinary Research. 38: 1111-1114.

6 Gutierrez C.B., Tascon R.I. \& Rodríguez-Barbosa J.I. 1993. Characterization of V factor-dependent organisms of the family Pasteurellaceae isolated from porcine pneumonic lungs in Spain. Comparative Immunology, Microbiology and Infectious Disease. 6: 123-130.

7 Kich J.D., Piffer I.A., Barcellos D.E.S.N., Guidoni A.L. \& Klein C.S. 1999. Utilização de um teste de ELISA polivalente para detecção de anticorpos para Actinobacillus pleuropneumoniae. Arquivo Brasileiro de Medicina Veterinária e Zootecnia. 51 (Suppl 5). 409-414.

8 Locatelli J.C., MachadoA. \& SilvaA.S. 1981. Ocorrência de pleuropneumonia suína devido a Haemophilus pleuropneumoniae. In: Resumos do VI Congresso Estadual de Medicina Veterinária (Gramado, Brasil). p.36-37.

9 Möller K. \& Killian M. 1990. V Factor-dependent members of the family Pasteurellaceae in the porcine upper respiratory tract. Journal of Clinical Microbiology. 28: 2711-2716.

10 Nielsen R. 1986. Serological characterization of Haemophilus pleuropneumoniae (Actinobacillus pleuropneumoniae) strains and proposal of a new serotype: 9. Acta Veterinaria Scandinavica. 27: 453-455.

11 Nielsen R., Andresen L.O. \& Plambeck T. 1997. Serological characterization of Actinobacillus pleuropenumoniae biotype 2 strains isolated from pigs in two Danish herds. Veterinary Microbiology. 54: 35-46.

12 Nielsen R. \& O'Conner P.J. 1983. Serological characterization of Haemophilus pleuropneumoniae strains and proposal of a new serotype: serotype 8. Acta Veterinaria Scandinavica. 25: 96-106.

13 Piffer I.A., Brito M.A.V.P. \& Brito J.R.F. 1987. Sorotipos de Haemophilus (Actinobacillus) pleuropneumoniae isolados de suínos no Brasil. Pesquisa Veterinária Brasileira. 7: 79-83.

14 Piffer I.A., Klein C. \& Fávero M.B.B. 1997. Caracterização bioquímica e sorológica de amostras de Actinobacillus pleuropneumoniae isoladas no Brasil. Arquivo Brasileiro de Medicina Veterinária e Zootecnia. 49 (Suppl 1). 123-129. 